inhibitor-1 and increasing severity of disease according to Dukes's stage, which is an established predictor of poor prognosis in patients with colorectal cancer.

We thank the RANX05 Colorectal Cancer Study Group for the collection of the plasma samples.

Contributors: HJN and NB had the original idea for and planned the study. HJN was also responsible for collecting the samples and patient data. FM established the database and participated in planning the clinical trial. JG-H developed the enzyme linked immunosorbent assay and analysed all the samples. IJC and HP were responsible for the statistical analyses of the data. The paper was written jointly by NB, KD, JG-H, OT-U, and HJN. HJN and NB are guarantors of the study.

Funding: This study received financial support from Glaxo Group Research, United Kingdom; the Kornerup Foundation; the Ingeborg Roikjer Foundation; the University of
Copenhagen; the Gerda and Aage Haench Foundation; and the Danish Cancer Society. Conflict of interest: None.

1 Danø K, Andreasen PA, Grøndahl-Hansen J, Kristensen P, Nielsen LS, Skriver L.Plasminogen activators, tissue degradation and cancer. Adt Cancer Res 1985;44:139-266.

2 Andreasen PA, Georg B, Lund LR, Riccio A, Stacey SN. Plasminogen activator inhibitors: hormonally regulated serpins. Mol Cell Endocrinol 1990;68:1-19.

3 Grøndahl-Hansen J, Christensen IJ, Rosenquist C, Brünner N, Mouridsen HT, Dan $\varnothing$ K, et al. High level of urokinase-type plasminogen activator and its inhibitor PAI-1 in cytosolic extracts of breast carcinomas are associated with poor prognosis. Cancer Res 1993;53:2513-21.

4 Casslen B, Bossmar T, Lecander I, Åstedt B. Plasminogen activators and plasminogen activator inhibitors in blood and tumour fluids of patients with ovarian cancer. Eur J Cancer 1994;30A:1302-9.

5 Meijer P, Pollet DE, Wauters J, Kluft C. Specificity of antigen assays of plasminogen activator inhibitor in plasma: Innotest plasminogen activator inhibitor-1 immunoassay evaluated. Clin Chem 1994;40:110-5. (Accepted 10 April 1997)

\title{
How much does relapse after one year erode effectiveness of smoking cessation treatments? Long term follow up of randomised trial of nicotine nasal spray
}

\author{
John A Stapleton, Gay Sutherland, Michael A H Russell
}

Institute of

Psychiatry, Tobacco

Research Section,

National Addiction

Centre, London

SE5 8AF

John A Stapleton,

senior lecturer

Gay Sutherland,

lecturer

Michael A H

Russell,

professor

Correspondence to:

Dr Stapleton

j.stapleton@iop.

bpmf.ac.uk

BMJ 1998;316:830-1

Recent research on treatments to stop smoking has focused almost entirely on nicotine replacement, and several meta-analyses testify to the efficacy of four delivery systems. ${ }^{1}$ Although the ultimate goal of treatment is lifelong cessation, few trials have published results of abstinence beyond one year. Little consideration has therefore been given to whether the treatment is effective in reducing the major health risks of smoking. This effect would become evident only after many years of abstinence. Our randomised trial showed that the use of nicotine nasal spray compared with a placebo spray was associated with more than double the number of abstainers at one year. ${ }^{2}$ We report the results from a longer term follow up to estimate the impact of relapse after one year on effectiveness.

\section{Subjects, methods, and results}

A total of 227 heavy smokers entered the trial; 116 were given the nicotine spray and 111 the placebo. Of these, 47 sustained abstinence from smoking for 1 year. They constituted the long term follow up group; 33 were in the nicotine group, 14 in the placebo group. Criteria for long term sustained abstinence were the same as for the first year. Since the follow up was completed mainly over a 2 month period, the time interval from randomisation varied according to when the smoker entered the trial over 15 months. Standard survival methods were used to

Results of long term follow up of randomised trial of nicotine nasal spray

\begin{tabular}{lccc} 
& $\begin{array}{c}\text { Nicotine } \\
\text { spray (n=116) }\end{array}$ & $\begin{array}{c}\text { Placebo spray } \\
(\mathbf{n = 1 1 1 )}\end{array}$ & $\begin{array}{c}\text { Difference in \% } \\
\text { (95\% Cl) }\end{array}$ \\
\hline$\%$ (No) who sustained abstinence to 1 year & $28.4(33)$ & $12.6(14)$ & 15.8 (5.6 to 26.1) \\
\hline Sustained abstinence to 3.5 years (\%) & 15.4 & 6.1 & $9.3(0.88$ to 17.4) \\
\hline Cumulative relapse between 1 and 3.5 years $(\%)^{*}$ & 45.9 & 52.1 & $-6.2(-41.0$ to 28.8) \\
\hline
\end{tabular}

*Kaplan-Meier estimates. analyse the data. Survival times of those who were not contacted beyond 1 year (3 subjects in the nicotine group, 2 in the placebo group) and those who had successfully given up were censored at their last follow up. The Kaplan-Meier method was used to estimate cumulative abstinence up to $3 \frac{1}{2}$ years.

Mean follow up period was 3 years 4 months (range $2 \frac{1}{2}$ to $4 \frac{2}{3}$ years) and was shorter by 21 days for the nicotine group. All observed relapses occurred within $3 \frac{1}{2}$ years. The table shows that the nicotine spray maintained an advantage over placebo up to $3 \frac{1}{2}$ years. Relapse after 1 year's abstinence was similar in the two groups and totalled 23\% at 2 years, $38 \%$ at 3 years and $48 \%$ (95\% confidence interval $32 \%$ to $64 \%$ ) at $3 \frac{1}{2}$ years. Although subjects had been recommended to use the spray for three months only, they were allowed to continue for 1 year. Of those remaining abstinent in the nicotine group, 19 used the spray for 1 year and 14 for $<1$ year (range 1-39 weeks). There was no difference in relapse after 1 year in the nicotine group between those who used the spray for 1 year and those who stopped earlier (difference 5\%, 95\% confidence interval $-33 \%$ to $43 \%)$.

\section{Comment}

Our results show that the spray is an effective aid to long term smoking cessation and that those who used the spray for 1 year had a similar relapse profile to those who stopped using it earlier. They also indicate substantial relapse after the time that most studies have completed their final follow up to assess treatment efficacy. Although the success ratio of active to placebo treatment (about 2.5) was unchanged by relapse, the absolute difference was reduced considerably, and hence the estimated number needed to treat to achieve each success was increased (from 6.3 to 10.8). 
Our relapse rate is similar to that in a trial of nicotine patches $(37 \% \text { between years } 1 \text { and } 3)^{3}$ and in a study using supportive counselling and nicotine gum for 5 years (40\% between years 1 and 5$).{ }^{4}$ High relapse rates after 1 year are also common in those not attending for treatment. A large general population survey estimated a relapse rate of $35 \%$ from non-validated self reports of the duration of abstinence. ${ }^{5}$

Success rates after 1 year or less of follow up substantially overestimate lifelong cessation after a single treatment episode.

Contributors: MAHR, GS, and JAS designed the original study, which GS conducted.JAS and GS designed the long term follow up phase, which GS conducted. JAS analysed the data and wrote the text of the paper.JAS and GS will act as guarantors for the paper.
Funding: Medical Research Council.

Conflict of interest: None.

Silagy C, Mant D, Fowler G, Lodge M. Meta-analysis on efficacy of nicotine replacement therapies in smoking cessation. Lancet 1994;343:139-42.

2 Sutherland G, Stapleton JA, Russell MAH, Jarvis MJ, Hajek P, Belcher M, et al. Randomised controlled trial of nasal nicotine spray in smoking cessation. Lancet 1992:340:324-9.

3 Mikkelsen KL, Tønnesen P, Nørregaard J. Three-year outcome of two-and three-year sustained abstainers from a smoking cessation study with nicotine patches. J Smok Rel Dis 1994;5:95-100.

4 Anthonisen NR, Connett JE, Kiley JP, Altose MD, Bailey WC, Buist AS, et al. Effects of smoking intervention and the use of an inhaled anticholinergic bronchodilator on the rate of decline of $\mathrm{FEV}_{1}$. JAMA 1994:272:1497-505.

5 US Department of Health and Human Services. Results from the NHANES-I population survey. In: The health benefits of smoking cessation: a report of the surgeon general. Washington, DC: DHHS, 1990. (Publication No CDC 90-8416.)

(Accepted 8 July 1997)

\title{
Management of deliberate self poisoning in adults in four teaching hospitals: descriptive study
}

\author{
Navneet Kapur, Allan House, Francis Creed, Eleanor Feldman, Trevor Friedman, Elspeth Guthrie
}

Deliberate self poisoning accounts for 100000 hospital admissions in England and Wales every year, and its incidence is increasing. ${ }^{1}$ One per cent of patients kill themselves in the year following attendance. ${ }^{2}$ Good services to manage deliberate self poisoning in general hospitals might therefore help to achieve the targets set out by the Health of the Nation strategy to reduce suicide rates. Existing services have not been planned coherently; the care provided by hospitals varying greatly, even in the same region. ${ }^{3}$ We assessed the management of self poisoning in four teaching hospitals in England by using standardised methods of notification.

\section{Subjects, methods, and results}

We prospectively identified all patients over 16 years of age who attended four teaching hospitals in Leeds, Leicester, Manchester, and Nottingham for deliberate self poisoning during 4 weeks (November to December 1996). We obtained data by examining computerised databases on wards and in the accident and emergency department, referral ledgers, accident and emergency notes, and copies of specialist assessments of deliberate self poisoning. We checked all inpatient data retrospectively against information on admission and discharge for deliberate self poisoning that we obtained from the patient administration system in each hospital. We collected demographic details of patients, along with details of substance dependence, previous overdoses, and contact with psychiatric services. We also recorded information on the management of the current episode of self poisoning.

During the study period 458 patients accounted for 477 hospital attendances for deliberate self poisoning; $223(49 \%)$ of these were women. The mean age of the patients was 30.9 years (SD 11.8 years); 65 (14\%) were dependent on alcohol or drugs, $177(39 \%)$ had taken a previous overdose, and $119(26 \%)$ were in contact with psychiatric services. These percentages and the substances ingested were similar across study centres. By contrast, there were striking variations in the management of episodes between study centres, with a fourfold difference in discharge rates from accident and emergency departments, and almost a twofold difference in the proportion of subjects receiving a specialist psychosocial assessment (table). In 220 out of 477 hospital attendances $(46 \%)$ the patient had no psychosocial assessment at any time during their hospital contact.

\section{Comment}

The average rate of patients with self poisoning presenting to hospital services in this study was 310 per 100000 population per year, which suggests that deliberate self poisoning accounts for 170000 hospital attendances in the United Kingdom annually. Yet services for this important problem remain in disarray. Striking variations in clinical practice were not accounted for by differences in patients' characteristics. We also discovered that, notwithstanding guidelines issued by the Department of Health, ${ }^{4}$ almost half of the patients in this study did not receive a specialist psychosocial assessment.

Our findings may reflect a high risk approach to intervention or a lack of consensus on the psychiatric management of self poisoning. ${ }^{5}$ We believe they probably reflect the low medical and psychiatric priority given to patients who have taken an overdose. A reduced number of beds means that medical staff are reluctant to admit patients who are judged to be at low physical risk and often seen as difficult and unrewarding. Meanwhile, psychiatric services are increasingly reserved for those with serious mental illness, a term which is not taken to include most cases of self poisoning. The current situation should not be allowed to continue because self poisoning represents a major social and clinical problem. At least, large scale intervention studies are required to inform practice and ensure that our management of deliberate self
Department of Liaison Psychiatry, Leeds General Infirmary, Leeds LS1 3EX

Navneet Kapur, research registrar Allan House, senior lecturer

Department of Psychiatry and Behavioural Sciences,

Manchester Royal Infirmary, Manchester M13 9WL

Francis Creed, professor Elspeth Guthrie, senior lecturer

Department of Psychological Medicine, John Radcliffe Hospital, Oxford OX3 9DU

Eleanor Feldman, consultant in liaison psychiatry

Leicester General Hospital, Leicester LE5 4PW

Trevor Friedman, consultant in liaison psychiatry

Correspondence to: Dr N Kapur, Department of Psychiatry and Behavioural Sciences,

Manchester Royal Infirmary, Manchester M13 9WL 